\title{
Spatial control of photoemitted electron beams using a microlens-array transverse-shaping technique
}

\author{
A. Halavanau, ${ }^{1,2}$ G. Qiang, ${ }^{3,4}$ G. Ha, ${ }^{5}$ E. Wisniewski, ${ }^{3}$ P. Piot, ${ }^{1,2}$ J. G. Power, ${ }^{3}$ and W. Gai ${ }^{3}$ \\ ${ }^{1}$ Department of Physics and Northern Illinois Center for Accelerator \& Detector Development, \\ Northern Illinois University, DeKalb, Illinois 60115, USA \\ ${ }^{2}$ Fermi National Accelerator Laboratory, Batavia, Illinois 60510, USA \\ ${ }^{3}$ Argonne Wakefield Accelerator, Argonne National Laboratory, Lemont, Illinois 60439, USA \\ ${ }^{4}$ Department of Engineering Physics, Tsinghua University, Beijing 100084, China \\ ${ }^{5}$ POSTECH, Pohang, Kyoungbuk, 37673, Korea
}

(Received 24 July 2017; published 26 October 2017)

\begin{abstract}
A transversely inhomogeneous laser distribution on the photocathode surface generally produces electron beams with degraded beam quality. In this paper, we explore the use of microlens arrays to dramatically improve the transverse uniformity of an ultraviolet drive-laser pulse used in a photoinjector. We also demonstrate a capability of microlens arrays to generate transversely modulated electron beams and present an application of such a feature to diagnose the properties of a magnetized beam.
\end{abstract}

DOI: 10.1103/PhysRevAccelBeams.20.103404

\section{INTRODUCTION}

Photoemission electron sources are widespread and serve as backbones of an increasing number of applications including high-energy particle accelerators, acceleratorbased light sources and ultrafast electron diffraction. For a given photoemission electron-source design, the electronbeam properties, and notably its brightness, are ultimately limited by the emission process and especially by the initial conditions set by the laser pulse impinging on the photocathode surface. Nonuniformities in the transverse electron-beam density result in transverse emittance dilution and in the development of intricate correlations. Producing and transporting a laser pulse while preserving a homogeneous transverse density is challenging and has been an active area of work [1]. In this paper, we investigate a simple technique capable of controlling the transverse shape of an ultraviolet (UV) laser pulse. The technique employs a pair of microlens arrays (MLAs) configured to transversely homogenize the laser pulse. MLAs are commonly employed as optical homogenizers for various applications [2-4]. In addition to its homogenizing capability, we also demonstrate that the proposed technique can also produce a periodic transverse pattern, consisting of a two-dimensional array of beamlets. This type of a beam could find application in beam-based diagnostics of accelerator beam lines, single-shot quantum-efficiency map measurement, and coherent light sources in the $\mathrm{THz}$ regime or at shorter wavelengths $[5,6]$. We establish the usefulness

Published by the American Physical Society under the terms of the Creative Commons Attribution 4.0 International license. Further distribution of this work must maintain attribution to the author(s) and the published article's title, journal citation, and DOI. of the MLAs to control the electron beam distribution in a series of experiments carried out at two accelerator beam lines available at the Argonne Wakefield Accelerator (AWA) facility [7]: the AWA drive beam (AWA-DB) injector was used to explore the homogenization process and the transport of modulated beam to $\sim 50 \mathrm{MeV}$ while the AWA witness-beam (AWA-WB) injector supported the multibeam application to measure the magnetization of a magnetized beam.

\section{OPTICAL PERFORMANCES OF THE MLA}

Qualitatively, the principle of the MLA lies in redistributing the incoming light intensity across the light beam spot. Typically, MLAs are arranged in pairs. After passing through the MLA assembly, the light rays are collected by a convex lens which focuses parallel rays from different light beamlets to a single point at the image plane. Under proper conditions, the process leads to transverse homogenizing of the beam; see Fig. 1. Therefore the MLA homogenization scheme is rather simple and appealing in the context of photocathode drive lasers.

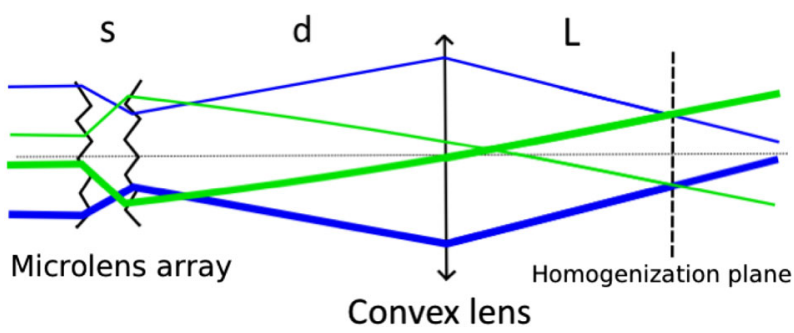

FIG. 1. Schematics of the microlens-array configuration. Initial intensity fluctuations in the beam (thin/thick ray) become evenly distributed at the homogenization plane. 
Alternatively, imaging the object plane of the single microlenses in the MLA with a convex lens produces a set of optical beamlets arranged as arrays with a pattern mimicking the microlens spatial distributions.

\section{A. ABCD formalism}

We first analyze the typical MLA setup diagrammed in Fig. 1 to derive a few salient features relevant to homogenization using the ABCD formalism [2]. We consider an initial ray to be characterized by the vector $\left(x_{0}, x_{0}^{\prime}\right)$, where $x_{0}$ and $x_{0}^{\prime} \equiv \frac{d x_{0}}{d z}$ are respectively the initial ray position and divergence (here $z$ represents the path length along the direction of the optical transport). As a simple example, we consider a rectangular array of microlens in the $(x, y)$ plane with an equal pitch in both transverse directions. Using the $\mathrm{ABCD}$ formalism, and considering that the ray is within the aperture $\rho$ of the lens with center located at $(x=m p, y=n p)$, we can describe the MLA with the linear transformation

$$
\begin{aligned}
\left(\begin{array}{c}
x_{1}-m p \\
x_{1}^{\prime}
\end{array}\right)= & \left(\begin{array}{cc}
1 & 0 \\
-1 / f_{2} & 1
\end{array}\right)\left(\begin{array}{cc}
1 & s \\
0 & 1
\end{array}\right) \\
& \times\left(\begin{array}{cc}
1 & 0 \\
-1 / f_{1} & 1
\end{array}\right)\left(\begin{array}{c}
x_{0}-m p \\
x_{0}^{\prime}
\end{array}\right),
\end{aligned}
$$

where $\left(x_{1}, x_{1}^{\prime}\right)$ is the ray vector after two MLA plates, $s$ is the spacing between two plates, $p$ is the array pitch, $f_{1}$ and $f_{2}$ are the focal lengths of the first and second microlens, respectively. It should be pointed out that the ray initial and final coordinates satisfy $\sqrt{\left(x_{0}-m p\right)^{2}+\left(y_{0}-n p\right)^{2}} \leq \rho$ where $n$ and $m$ are integers that specify the position of each microlens in terms of the pitch. Then, the output ray from the MLA setup can be further propagated to the homogenization plane as

$$
\left(\begin{array}{l}
x_{h} \\
x_{h}^{\prime}
\end{array}\right)=\left(\begin{array}{ll}
1 & L \\
0 & 1
\end{array}\right)\left(\begin{array}{cc}
1 & 0 \\
-1 / F & 1
\end{array}\right)\left(\begin{array}{ll}
1 & d \\
0 & 1
\end{array}\right)\left(\begin{array}{l}
x_{1} \\
x_{1}^{\prime}
\end{array}\right),
$$

where $\left(x_{h}, x_{h}^{\prime}\right)$ is the ray vector at the homogenization plane, $d$ is the distance between the convex lens and the MLA, $F$ is the focal length of the convex lens and $L$ is the distance to the homogenization plane.

From the formalism above one can deduce a few useful expressions. First, we specialize to the case when the two MLAs are identical $\left(f_{1}=f_{2}=f\right)$ and located in the object plane of the lens $(L=F)$. We further assume that there is no cross talk between the microlens and their transformation only affects rays within a finite aperture smaller than the array pitch $\sqrt{\left(x_{0}-m p\right)^{2}+\left(y_{0}-n p\right)^{2}} \leq p / 2$. Under these assumptions, we find the diameter of the image at the homogenization plane to be

$$
D_{h} \approx \frac{F p}{f^{2}}(2 f-s)
$$

in the limit of small ray divergence (as indicated by the independence of the equation on $d$ ). For practical purposes, we also calculate the diameter of the beam at the lens plane to be

$$
A_{F} \approx \frac{d p}{f^{2}}(2 f-s) .
$$

This equation is useful to estimate the required aperture.

In practice, the assumption $L=F$ might be challenging to satisfy. In such cases, the following expression is useful to find the beam size at a given location $L$ with respect to the lens:

$$
D(L) \approx \frac{p L}{f^{2}}(2 f-s)+\frac{d p(2 f-s)}{f^{2}} \frac{F-L}{F} .
$$

If $L \approx F$ the resulting image remains homogenized due to the finite size of the Airy disk. Moving away from the focal plane increases the density modulations and eventually yields an array of beamlets.

\section{B. Optical transport design}

Photoinjector setups often incorporate relatively long (multimeter scale) optical transport lines. A commonly used imaging setup, known as $4 f$-imaging, is challenging to implement in the present case as it would require some of the lenses to be located inside the vacuum chamber, as the "imaging" plane has to be much farther downstream than the "object" plane upstream. However, imaging can be achieved in numerous ways while accommodating the constraints related to MLAs (limited apertures, available focal lengths, etc.). To construct the appropriate optical line we impose the vector of a ray in the homogenization plane $\left(x_{h}, x_{h}^{\prime}\right)$ to be transported to a downstream imaging plane $\left(x_{I}, x_{I}^{\prime}\right)$ via

$$
\left(\begin{array}{c}
x_{I} \\
x_{I}^{\prime}
\end{array}\right)=\mathbf{M}\left(\begin{array}{c}
x_{h} \\
x_{h}^{\prime}
\end{array}\right), \quad \text { with } \quad \mathbf{M}=\left(\begin{array}{cc}
\mathcal{M} & 0 \\
0 & 1 / \mathcal{M}
\end{array}\right),
$$

where the magnification $\mathcal{M}$ is set to 1 for one-to-one imaging. Constructing an optical system with the required M yields four equations; an additional constraint comes from the total length of the imaging transport. Therefore, the problem has five unknowns in total with some flexibility in available lenses. Hence, it is possible to construct a four-lens solution with distances between lenses as free parameters to make the corresponding system of linear equations well defined.

The simulation of such a four-lens system was accomplished with a simple ray-tracing program where an initial set of optical rays was distributed according to a twodimensional Gaussian distribution in the $\left(x, x^{\prime}\right)$ optical trace space. The optical layout of the laser transport downstream of the MLA is depicted in Fig. 2(a): it includes four cylindrical-symmetric lenses, an optical window that allows for the laser beam to be injected in an ultrahigh-vacuum area and an in-vacuum metallic mirror that directs the laser beam 

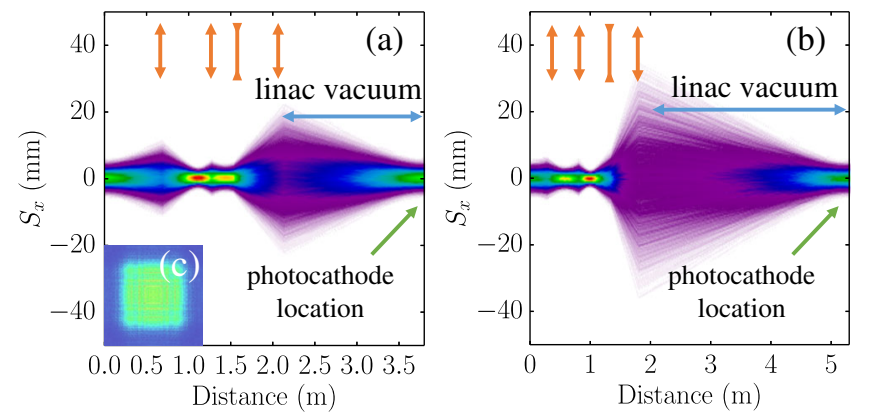

FIG. 2. False color ray-tracing distribution of a four-lens optical line capable of imaging the homogenized beam on the photocathode surface. The configuration in (a) and (b) corresponds respectively to the AWA witness-beam (AWA-WB) and drivebeam (AWA-DB) electron-source setups. The lens types and locations are shown as red arrows. The inset (c) gives the intensity distribution simulated using the vectorial-diffraction program SRW for a $5 \times 5$ rectangular MLA.

on the cathode surface. The resulting evolution of the beam size along the transport downstream of the MLA and up to the photocathode is displayed in Figs. 2(a) and 2(b) for the two rf-gun configurations associated with the AWA-WB (a) and AWA-DB (b) beam lines, respectively. For both setups, the large beam size produced at the location of the last optical lens demands a large-aperture lens. The beam size downstream gradually decreases until it reaches its target transverse size on the photocathode surface [2 $\mathrm{mm}(\mathrm{rms})]$. The in-vacuum mirror located close to the last optical transport lens can be another limiting aperture of the optical system and generally results in beam losses. For the two cases reported in Figs. 2(a) and 2(b) the MLA-tocathode transmission due to the finite geometric aperture, window transmission coefficient, losses in the lenses and mirrors was computed to be $57 \%$ and $43 \%$.

The designs presented in Fig. 2 were also simulated with the SYNCHROTRON RADIATION WORKSHOP (SRW software [8]) which is based on Fourier optics and readily includes a wave-propagation treatment of the laser transport; see the Fig. 2(c) inset. It confirmed that diffraction effects in the setup are negligible compared to transmission losses in the optical system.

Finally, transverse shot-to-shot jitter in the transverse distribution displayed in Fig. 3 (left) would result in charge fluctuations if the laser beam was collimated by an iris upstream of the MLA. To improve the stability of the laser intensity we introduced a two-lens beam reducer in front of the MLA.

\section{Optical measurements}

To evaluate the performance of the proposed scheme, we tested the MLA assembly using the photocathode drive laser of the AWA-DB photoinjector [7]. The input UV $(\lambda=248 \mathrm{~nm})$ laser pulse was obtained from frequency tripling of an amplified IR pulse originating from a Ti:Sp laser system. Downstream of the frequency tripler, the UV pulse is further amplified in a two-pass excimer amplifier before transport to the accelerator vault. The setup displayed in Fig. 1 was followed by the optical transport line shown in Fig. 2(b). A calibrated UV-sensitive screen with associated CCD camera mounted downstream of the setup provided a direct measurement of the transverse laser distribution.

To gain confidence in the performances of the MLA setup, we first investigated the impact of a nonperfectly collimated incoming laser beam. The homogenization can still be achieved even if the incoming beam has a small divergence. However, there is a critical value of beam divergence $\tan \theta=p / 2 f$ that causes destructive interference after the MLA and results in light loss [9].

The beam size provided by Eq. (3) was used in the optical relay setup and Eq. (4) to infer the aperture value of the convex lens located upstream of the MLA setup. Note that the convex lens in the experimental setup should be placed at the distance $D>F$ from the array, where $F$ is the focal length of the lens.

The setup was employed to demonstrate the homogenization process and quantify its performance. The nominal UV laser pulse was used as a starting condition; see Fig. 3(a). The inhomogeneity of the transverse distribution can be quantified using the spatial Fourier transform [10]. Correspondingly, we consider the digitized image $I(x, y)$ associated with the transverse laser distribution and compute its two-dimensional (2D) Fourier transform $\tilde{I}\left(k_{x}, k_{y}\right)$ using the fast-Fourier-transform (FFT) algorithm available in the PYTHON's NUMPY toolbox [11]. Here $k_{x}, k_{y}>0$ are the spatial wave numbers respectively associated to the horizontal and vertical directions. In order to simplify the comparison we further introduce the transverse bunching factor (TBF) as the one-dimensional Fourier transform $\tilde{I}_{x}\left(k_{x}\right)=\int_{0}^{+\infty} \tilde{I}\left(k_{x}, k_{y}\right) d k_{y}$ along the horizontal axis [a similar definition holds for the vertical axis $\left.\tilde{I}_{y}\left(k_{y}\right)\right]$. Figures $3(\mathrm{~d})$ and $3(\mathrm{~g})$ respectively correspond to the $2 \mathrm{D}$ Fourier transform and the projection along the horizontal wave number $k_{x}$ axis associated to the laser distribution displayed in Fig. 3(a). It displays typical microstructures observed in previous runs at AWA, and the corresponding spectrum displays some small modulations at low frequencies with most of the spectral content below $k_{i}<5 \mathrm{~mm}^{-1}$. It should be noted that the excessive beam distortion observed in Fig. 3(a) is the result of beam filamentation as the highenergy UV pulse propagates in the 20 -m open-air optical transport system from the laser room to the accelerator vault.

The MLA can be arranged to form a transversely modulated laser distribution; the spectrum indicates a $\mathrm{TBF}$ at frequencies larger than the characteristic frequency associated to the total beam size; see Figs. 3(b), 3(e), and 3(h). When the MLA setup is configured to homogenize the beam [see Fig. 3(c)], the Fourier transform indicates that, although the low frequency modulations seen in the original beam are suppressed, high-frequency modulations are present for $k_{x}>12 \mathrm{~mm}^{-1}$. These modulations have a 

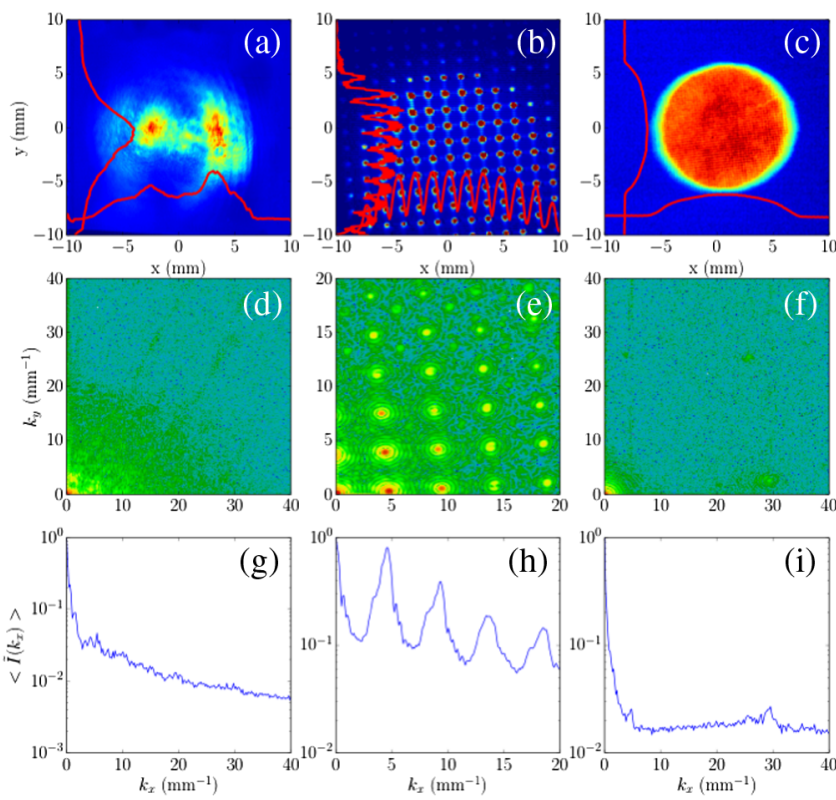

FIG. 3. Measured UV laser without MLA (left column) and with MLA setup to produce beamlets (middle column) or as a homogenizer (right column). The upper, middle and lower rows respectively correspond to the laser transverse density distribution, its 2D FFT, and the projected spectrum along the horizontal spatial frequency $k_{x}$.

TBF amplitude on the order of $10^{-2}$ and correspond to very small modulation wavelength $(<0.5 \mathrm{~mm})$ barely observable on the distribution; see Fig. 4.

Figure 4 compares the projected horizontal Fourier spectra for four cases of MLA configurations. Each spectrum is obtained by averaging five measurements taken after $f=250 \mathrm{~mm}$ convex lens at 250,275 and $325 \mathrm{~mm}$ to study the off-focal modulation and pattern formation. The latter figure confirms that in the homogenization regime, the MLA setup significantly improves the image spectrum by suppressing the low-frequency modulations present in the initial laser distribution.

Finally, we quantify the laser power loss in the devised setup. The MLA plates employed in our series of

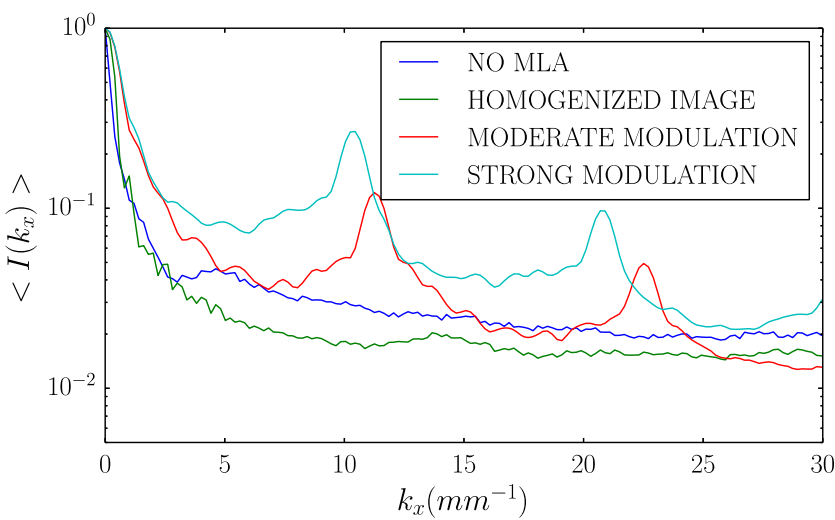

FIG. 4. Horizontal transverse bunching factor $\tilde{I}\left(k_{x}\right)$ for different positions of the lens located downstream of the MLA setup. The blue trace corresponds to no MLA case. The green, red, and turquoise traces respectively correspond to the convex lens located 250, 275 and $325 \mathrm{~mm}$ downstream of the MLA assembly.

experiments do not have any UV antireflection (AR) coating, hence the power loss was $\sim 5 \%$ per surface totaling $\sim 20 \%$ for the two MLAs. Additionally, the AR-coated UV lenses introduce a power loss of $\sim 2 \%$ per lens. In our optical setup the laser energy was measured to be $4.2 \pm 0.1$ and $2.5 \pm$ $0.1 \mathrm{~mJ}$ respectively upstream and downstream of the MLA setup including the first convex and four transport lenses. Such a measurement indicates an energy transmission of $\sim 60 \%$ which could most likely be improved in an optimized setup.

\section{APPLICATION OF THE MLA AS A LASER HOMOGENIZER}

The first set of experiments focused on demonstrating the simple homogenization technique to improve the emittance of an accelerator. The experiment was performed in the AWA-BD accelerator diagrammed in Fig. 5. In brief, the transversely manipulated UV laser pulse impinges on a high-quantum efficiency cesium telluride $\left(\mathrm{Cs}_{2} \mathrm{Te}\right)$ cathode located in the L-band (1.3-GHz) rf gun to produce $7 \mathrm{MeV}$

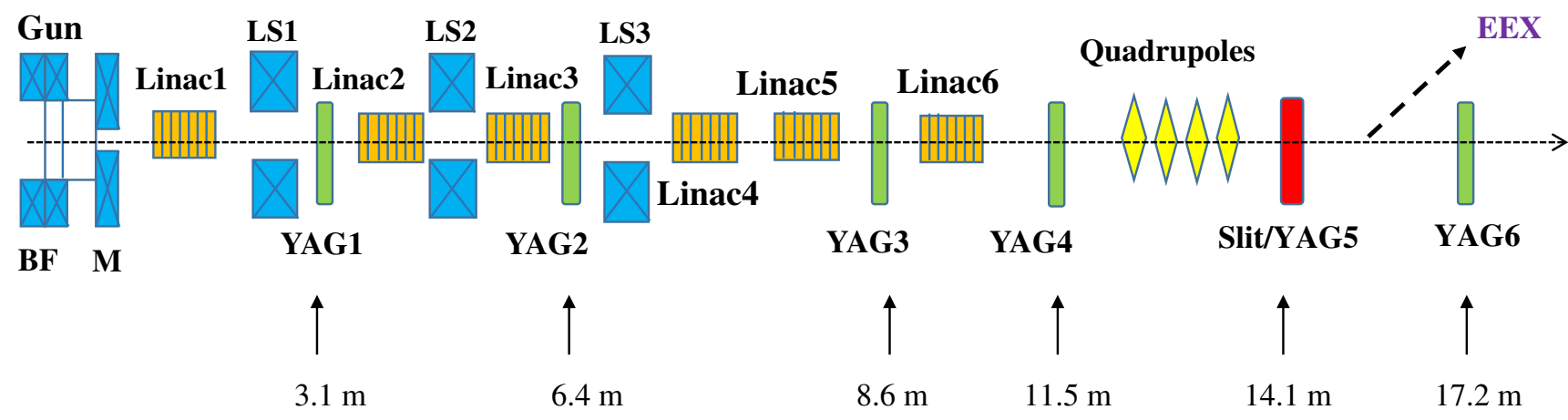

FIG. 5. Overview of the AWA-DB beam line only showing elements relevant to the performed experiment. Bucking-focusing (BF) and matching (M) solenoids were adjusted to image the beam on YAG screens. Linac solenoids (LS) and quadrupoles were turned off during the experiment. The positions of the YAG viewers are denoted in meters. The energy gain of one accelerating cavity (linac) is $10 \mathrm{MeV}$. EEX label marks the separate double-dogleg beam line for emittance exchange experiments. 
electron bunch. The electron bunches are then further accelerated in an L-band normal conducting cavities up to $75 \mathrm{MeV}$. For a detailed description of the facility, the reader is referred to Ref. [7]. The rf gun is surrounded by three solenoidal lenses referred to as bucking, focusing and main solenoids. The bucking and focusing solenoid have opposite polarity and are ganged to ensure the axial magnetic field on the photocathode vanishes. Several YAG: Ce scintillating screens (YAG in Fig. 5) are available to measure the beam transverse density along the accelerator beam line.

\section{A. Beam dynamics simulations}

We carried out several simulations using the beamdynamics program General PARTicle Tracker (GPT) [12] to explore the impact of the MLA-homogenized beam on the resulting emittance. Transverse inhomogeneities on the laser distribution at the photocathode surface result in similar inhomogeneities on the photoemitted electron bunch distribution. These imperfections result in asymmetric space-charge forces and eventually yield phasespace dilution that ultimately degrades the beam emittances [13]. Therefore the homogenized laser beam is expected to improve the beam transverse emittance.

The initial macroparticle distribution was produced using a Monte-Carlo generator using the measured transverse distribution of the laser similar to Ref. [14]. The temporal laser distribution was taken to be Gaussian with rms duration $\sigma_{t}=2.5 \mathrm{ps}$, consistent with streak camera measurements. The momentum of the macroparticle assumes an excess kinetic energy of $0.5 \mathrm{eV}$ as typically considered for $\mathrm{Cs}_{2} \mathrm{Te}$ cathodes [15]. We considered the nominal and homogenized laser distribution respectively shown in Figs. 3(a) and 3(c). To ensure an accurate comparison, the total charge for both cases of distributions was set to $1 \mathrm{nC}$. Likewise, the rms transverse sizes of the distribution was fixed to $\sigma_{c}=2 \mathrm{~mm}$ along both the horizontal and vertical directions. The simulations demonstrate that the beam transverse emittances are reduced by more than a factor $\sim 2$ for the case of the homogenized laser distribution; see Table I.

\section{B. Transverse emittance measurements}

The experimental verification of the benefits of homogenizing the laser distribution was accomplished using the measured distribution of Figs. 3(a) and 3(c). For the homogenized distribution displayed in Fig. 3(c), a circular iris was used to clip the laser distribution and ensure it had the same rms value as in Fig. 3(a) $\sigma_{c}=2 \pm 0.2 \mathrm{~mm}$. The resulting electron beam was transported through the nominal AWA-DB beam line and accelerated to a final mean momentum $p=48 \pm 0.5 \mathrm{MeV} / c$. The corresponding electron-beam transverse distributions measured at YAG5 are compared in Figs. 6(a) and 6(b). The distribution originating from the initial profile presents beam asymmetry and $x-y$
TABLE I. Comparison between measured and simulated beam parameters at YAG5 for $Q=1 \pm 0.1 \mathrm{nC}$. The experimental setup only allowed for the vertical normalized emittance to be measured. The parameters are all given as rms quantities and correspond to the distributions shown in Fig. 6.

\begin{tabular}{|c|c|c|c|}
\hline \multicolumn{4}{|c|}{ Experimental conditions } \\
\hline Parameter & No MLA & MLA & Units \\
\hline \multicolumn{4}{|c|}{ Simulation with GPT } \\
\hline Momentum $\langle p\rangle$ & 48 & 48 & $\mathrm{MeV} / c$ \\
\hline$\sigma_{x}$ & 3.6 & 3.8 & $\mathrm{~mm}$ \\
\hline$\sigma_{y}$ & 4.4 & 3.9 & $\mathrm{~mm}$ \\
\hline$\sigma_{x}^{\prime}$ & 4.6 & 1.7 & $\times 10^{-2} \mathrm{mrd}$ \\
\hline$\sigma_{y}^{\prime}$ & 3.1 & 1.5 & $\times 10^{-2} \mathrm{mrd}$ \\
\hline$\varepsilon_{x}$ & 15.6 & 6.1 & $\mu \mathrm{m}$ \\
\hline$\varepsilon_{y}$ & 12.8 & 5.5 & $\mu \mathrm{m}$ \\
\hline \multicolumn{4}{|c|}{ Measurement } \\
\hline Momentum $\langle p\rangle$ & $48 \pm 0.5$ & $48 \pm 0.5$ & $\mathrm{MeV} / c$ \\
\hline$\sigma_{x}$ & $4.4 \pm 0.2$ & $4.0 \pm 0.2$ & $\mathrm{~mm}$ \\
\hline$\sigma_{y}$ & $5.2 \pm 0.2$ & $3.7 \pm 0.2$ & $\mathrm{~mm}$ \\
\hline$\sigma_{y}^{\prime}$ & $4.2 \pm 1.3$ & $3.3 \pm 1.0$ & $\times 10^{-2} \mathrm{mrd}$ \\
\hline$\varepsilon_{y}$ & $20.5 \pm 7.4$ & $11.6 \pm 4.3$ & $\mu \mathrm{m}$ \\
\hline
\end{tabular}

coupling; see Fig. 6(a). In contrast, the homogenized distribution is cylindrically symmetric and does not show any coupling; see Fig. 6(b). To further quantify the improvement we measured the beam vertical emittance using the slit technique. A $100-\mu \mathrm{m}$ wide horizontal slit was inserted at YAG5 and the transmitted beamlet was observed $3.1 \mathrm{~m}$ downstream at YAG6 thereby providing the beam divergence $\sigma_{y}^{\prime}$. Such a measurement together with the vertical beam-size measurement at YAG5, $\sigma_{y}$, provides an estimate of the normalized vertical emittance via $\varepsilon_{y}=\beta \gamma \sigma_{y} \sigma_{y}^{\prime}$, where $\beta \approx 1$ and $\gamma=93.9$. The reported emittance is the core emittance and does not fully characterize the beam transverse phase space. The resulting beamlet distributions at
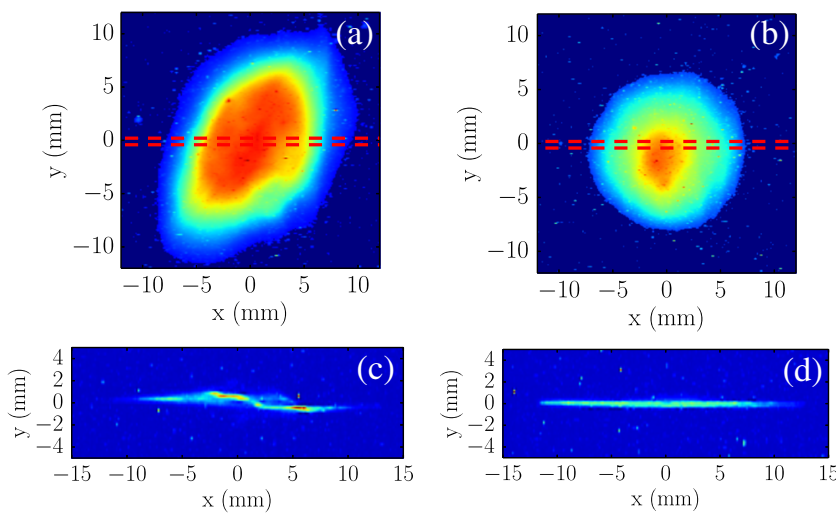

FIG. 6. Beam transverse distribution at YAG5 [(a), (b)] and associated distribution of the beamlet transmitted through a horizontal slit located at YAG5 location and measured at YAG6 [(c), (d)]. The set of images [(a), (b)] [respectively (c), (d)] corresponds to the case when the MLA was retracted [respectively inserted] from the laser-beam path. The horizontal dashed line in (a) and (b) represents the aperture of the slit. 
YAG5/6 are shown in Figs. 6(c) and 6(d) and the measured divergence and resulting normalized emittance compared in Table I. The value of $\varepsilon_{y}$ for the "No MLA" case was measured to be $20.5 \pm 7.4 \mu \mathrm{m}$ and for the "MLA" case $11.6 \pm 4.3 \mu \mathrm{m}$. The emittances are comparable to the values simulated with GPT (Table I) and indicate a factor $\sim 2$ improvement when the homogenized laser beam is employed. The relatively large error bars in Table I are due to hardware uncertainty (mostly the slit width). It should be noted that the errors between the two measurements are correlated, i.e. the uncertainty yields to the upper (respectively lower) value for simultaneously the "MLA" and "No MLA" measurements. Hence, the use of the MLA to homogenize the laser transverse distribution significantly improves the electron beam quality.

\section{PRODUCTION AND TRANSPORT OF MULTIBEAM ARRAYS}

In this section we explore the application of the MLA to generate two-dimensional arrays of beamlets. Such a multibeam array may serve as a beam-based diagnostic tool, e.g., to investigate nonlinearities of an externally
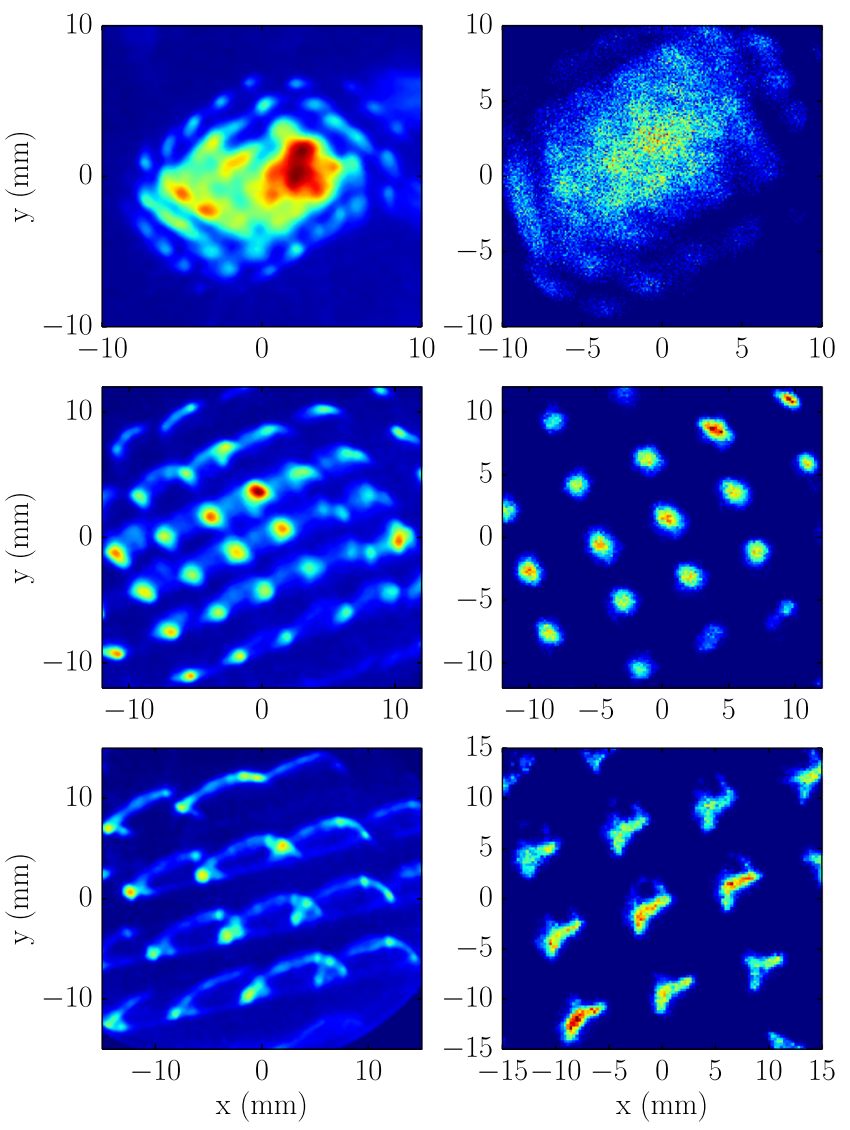

FIG. 7. Measured (left) and simulated (right) $Q=100 \mathrm{pC}$ electron-beam distribution at YAG1 when the UV laser pulse is modulated with the MLA array. The rows correspond to different matching-solenoid current settings of 215 A (upper row), $230 \mathrm{~A}$ (middle row) and $290 \mathrm{~A}$ (lower row). applied electromagnetic field or measure transfer matrices of beam line elements. In this section, we study the preservation of the initial laser modulation during the photoemission processes and subsequent acceleration in an rf gun and linear accelerator.

\section{A. Beam dynamics simulations}

Using the particle tracking codes GPT and IMPACT-T [16] we performed simulation of the AWA-DB rf-gun beam dynamics. The preservation of the modulation is affected by space-charge forces which play a dominant role in the beam dynamics in the vicinity of the cathode and in the rf gun. Given the multiscale nature of our problem, the spacecharge forces are computed with a Barnes-Hut (BH) algorithm [17] available in GPT. A similar algorithm was successfully tested in recent studies $[18,19]$.

The measured transversely modulated laser distribution at the photocathode location [similar to Fig. 3(b)] was used to generate the input macroparticle distribution for our numerical simulations. The resulting electron-beam beamlet pattern provided a benchmark for the GPT model with the experimental measurement downstream at low energy (downstream of rf gun); see Fig. 7. The beam data and numerical simulations were recorded downstream of the gun using a YAG:Ce scintillating screen (YAG1 in Fig. 5) located at $z=3.1 \mathrm{~m}$ from the photocathode surface. The beam energy was $7 \pm 0.5 \mathrm{MeV}$ and total bunch charge was set to $150 \mathrm{pC}$. The general qualitative agreement in the pattern rotation between simulation and measurement provided confidence in the GPT model; see Fig. 7.

\section{B. Multibeam formation downstream of the rf gun}

A subsequent experiment investigated the formation of a beamlet array downstream of the rf gun for various
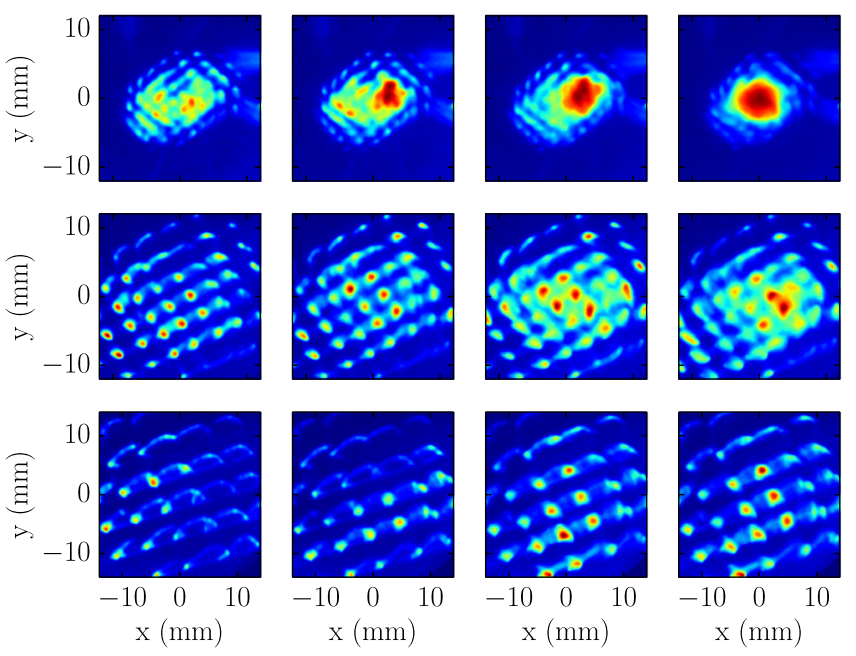

FIG. 8. False color measured $7 \mathrm{MeV}$ electron beam patterns for various matching solenoid current setting and charge. From left to right: $Q=60,80,100$, and $120 \mathrm{pC}$. The images from top to bottom correspond to matching-solenoid currents of 215, 240, and $270 \mathrm{~A}$. 
operating points of the photoinjector. The incoming laser spot size on the MLA array was chosen to yield an $8 \times 8$ beamlet array. The photoemitted electron beam was observed on YAG1. Figure 8 displays a sequence of beam distributions recorded at YAG1 for different settings of the focusing-bucking and matching solenoids. Note that, due to the surface space charge effects, the charge associated with each beamlet, and therefore the total maximum charge of the patterned beam, is limited. The total maximum charge of the patterned beam was measured to be approximately $\sim 15 \mathrm{nC}$ corresponding to an average charge of $\sim(15 \mathrm{nC}) /(8 \times 8) \simeq 200 \mathrm{pC}$ per beamlet.

The resulting electron beamlet formations pictured on Fig. 8 were analyzed using the same Fourier analysis as the
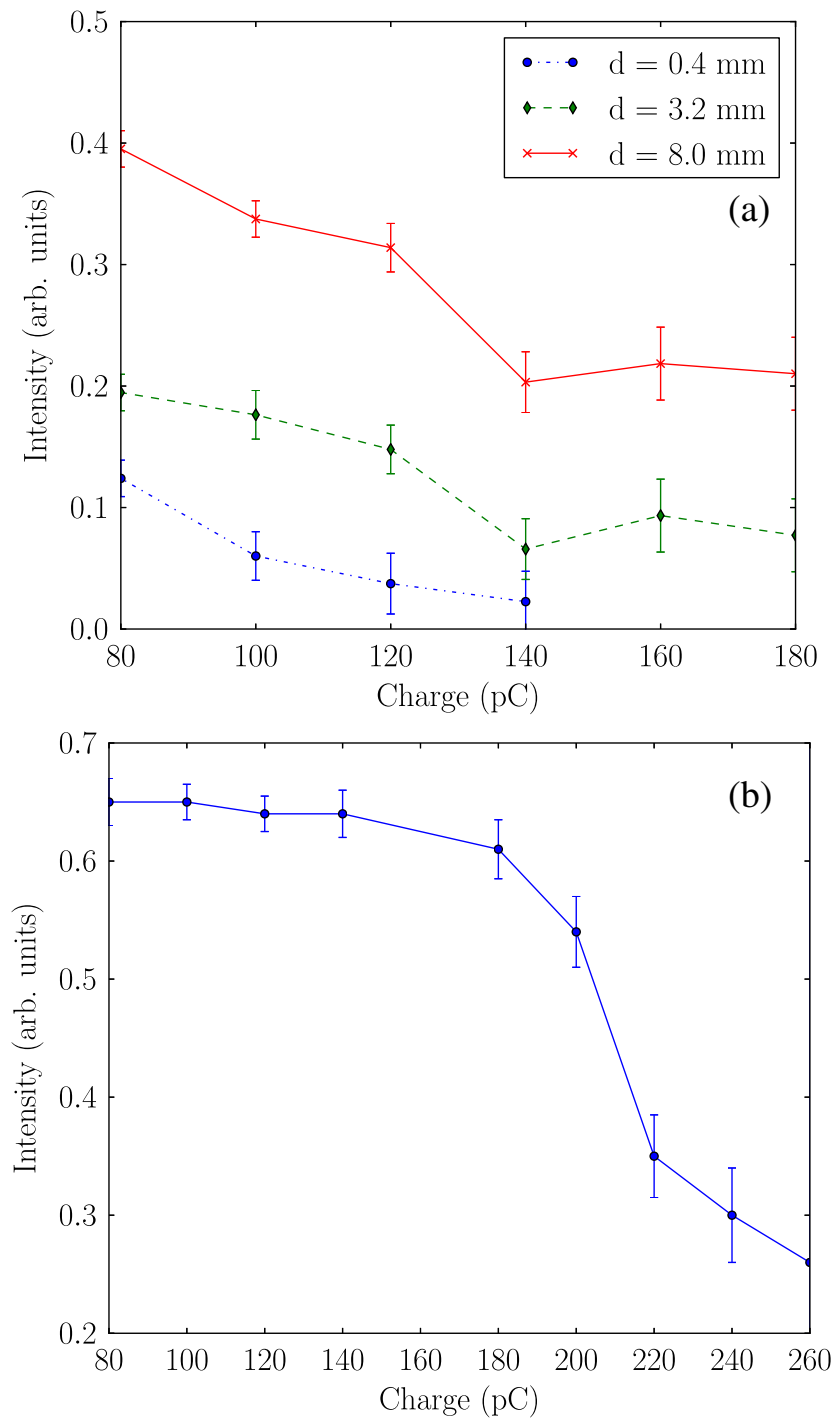

FIG. 9. TBF evaluated at its lowest-frequency maximum versus bunch charge for the three cases of solenoid settings displayed in Fig. 8 with corresponding beamlet spacing $d$ (a) and for the case of a solenoid field of 290 A with associated beamlet spacing of $d=10 \mathrm{~mm}(\mathrm{~b})$. one used in Sec. II C for the UV-laser images. Figure 9(a) summarizes the evolution of the TBF at its lowest-frequency maximum versus total bunch charge for different matching solenoid settings. The average beamlet separation $d$ changes from 0.4 to $8 \mathrm{~mm}$ at the YAG1 location with the matching solenoid current increased from 215 to 270 A. Figure 9(b) gives the evolution of the TBF for the case of $d=10 \pm 0.4 \mathrm{~mm}$. One can see the modulation is fully determined by solenoid imaging at charges of $Q<180 \mathrm{pC}$ but is significantly suppressed at higher charges.

\section{Multibeam acceleration to $48 \mathrm{MeV}$}

The modulation introduced on the cathode propagated and preserved through the beam line up to the transverseto-longitudinal emittance-exchange (EEX) beam line entrance; see Fig. 10. There should be no strong focusing applied along the low-energy beam line as closely spaced beamlets produce strong distortion as explored in Ref. [14]. At medium energy, the transverse space-charge force is significantly decreased and therefore not expected to impact the multibeam dynamics. In order to avoid a tight waist at low energy we used the linac solenoid LS1 (see Fig. 5) to image the beamlet pattern directly on the YAG5 screen located 14-m downstream of the photocathode surface and just prior to the EEX beam line. At this location, the beam energy was measured to be $48 \mathrm{MeV}$. Figure 10 shows the beam distribution at YAG5 for different bunch charges. The typical beamlet separation (center to center) was on the order of $\sim 3 \mathrm{~mm} \pm 0.3 \mathrm{~mm}$. Such a distribution could be further manipulated using a telescope composed of four quadrupole magnets to generate a train of short bunches along the temporal axis downstream of the EEX beam line [20]. Such a bunch train could possibly support the generation of $\mathrm{THz}$ radiation using, e.g., coherent transition radiation, or the resonant excitation of wakefields in a wakefield structure such as a dielectric-lined waveguide [21]. The coupling at YAG5 could be removed by mounting the MLA assembly on a rotatable mount. Likewise, the coupling could be exploited
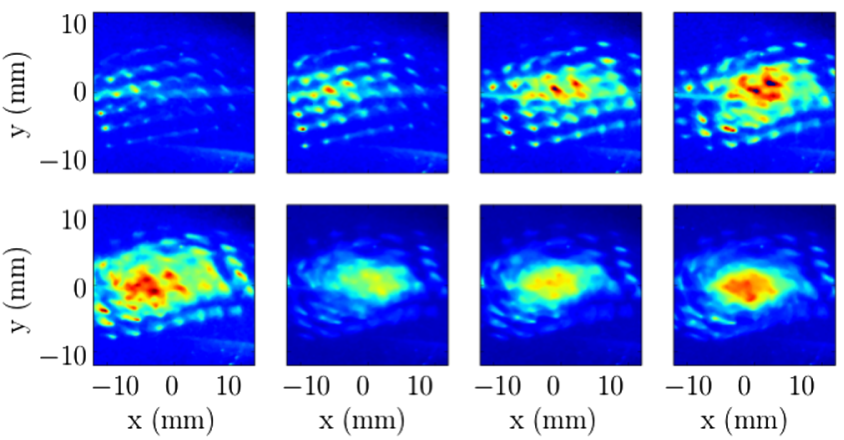

FIG. 10. False color measured $48 \mathrm{MeV}$ electron beam patterns for various charges. From left to right and top to bottom: $Q=60$, $100,200,300,400,500,600,700 \mathrm{pC}$ with a matching-solenoid current of 240 A. 
by selecting an angle such that a smaller projected separation along the horizontal axis could be achieved. Such a configuration would provide a knob to continuously vary the beamlets separation (e.g., and THz-enhancement frequency) downstream of the EEX beam line.

\section{GENERATION OF MAGNETIZED MULTIBEAMS}

In this section, we present an application of the patterned electron beam formed by the MLA setup as a beam-based diagnostic tool for inferring the residual axial magnetic field at the photocathode plate by measuring the value of canonical angular momentum (CAM). One of the main benefits of the MLA setup is that a beam line device is not needed to impose transverse structure on the beam.

As an example, we consider the AWA-DB beam line diagrammed in Fig. 11. In brief, the AWA-WB accelerator incorporates an L-band $1+\frac{1}{2}$ rf gun with a $\mathrm{Mg}$ photocathode on its back plate. The gun is surrounded by a bucking and focusing solenoids, nominally powered to yield a vanishing axial magnetic field $B_{0 z}$ at the photocathode surface. The solenoids can be tuned and provide a nonvanishing $B_{0 z}$ at the cathode. The bucking and focusing solenoids have the opposite polarity, but can be configured to provide significant field $\left(B_{0 z} \sim 0.1 \mathrm{~T}\right)$ on the photocathode and generate magnetized beam.

\section{A. Magnetized beams}

According to Busch's theorem the total canonical angular momentum of an electron in a cylindrically symmetric magnetic field is conserved and given by [22]

$$
L=\gamma m_{e} r^{2} \dot{\theta}+\frac{1}{2} e B_{z}(z) r^{2}+\mathcal{O}\left(r^{4}\right),
$$

where $(r, \theta, z)$ refers to the electron transverse position in the cylindrical coordinate system, $m_{e}$ is the electron mass and $B_{z}(z)$ is the axial magnetic field profile.

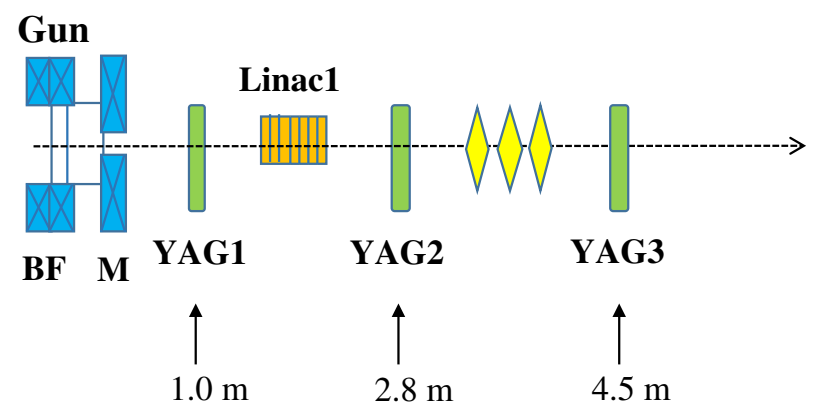

FIG. 11. Overview of the AWA-WB beam line. The bucking (B) and focusing (F) solenoidal lenses can be set up to produce CAM-dominated beams. The positions of the YAG viewers are denoted in meters.
The conservation of the CAM $L$ from Eq. (6) yields the mechanical angular momentum (MAM) of the beam in the magnetic-field-free zone to be

$$
|\boldsymbol{L}|=\gamma m_{e}\left|\boldsymbol{r} \times \frac{d \boldsymbol{r}}{d t}\right|=\frac{1}{2} e B_{0 z} r_{0}^{2},
$$

where $B_{0 z}$ is the field at the cathode surface, $r_{0}$ and $r$ are respectively the electron radial coordinate on the photocathode surface and at a downstream magnetic-field-free location. In single-particle and paraxial approximations, the CAM is fully transferred to MAM.

\section{B. Method to measure $\mathcal{L}$}

We now consider the multibeam laser distribution discussed in Sec. IV impinging on a photocathode immersed in an axial magnetic field. The resulting electron beam, composed of multiple beamlets, is born with CAM and will therefore undergo a similarity transformation (in the presence of a axisymmetric external focusing) of the form

$$
\left(\begin{array}{l}
x \\
y
\end{array}\right)=[k+R(\theta)]\left(\begin{array}{l}
x_{c} \\
y_{c}
\end{array}\right),
$$

after exiting the magnetic-field region. In the previous equation the subscript ${ }_{c}$ corresponds to the spatial coordinates on the cathode surface, $k$ is a scalar and $R(\theta)$ is the $2 \times 2$ matrix associated to a rotation with angle $\theta$. We define the relative rotation angle as $\psi=\theta_{2}-\theta_{1}$, where $\theta_{1,2}$ is the angle of rotation at two axial positions, then the value of MAM can be computed as [23]

$$
L=\frac{p_{z}}{D}\left[\left(\frac{n}{2} a_{1}\right)\right]^{2}(M \sin \psi),
$$

where $p_{z}$ is the axial momentum, $n$ is a number of beamlets, $a_{1}$ is the separation between beamlets at the first viewer, and $M=a_{2} / a_{1}$ is the magnification factor between second and first viewer. Relating the latter equation to Eq. (7) provides the value of the magnetic field on the cathode $B_{0 z}$.

\section{Electron beam experiment}

A proof-of-principle electron beam experiment was performed at the AWA-WBA beam line. A $12 \times 12$ laser beamlet pattern with rms duration of 6 ps was formed by using the technique from Sec. IV. The $\sim 5-\mathrm{MeV}$ beam out of the rf gun was further accelerated using the L-band linac to $\sim 10 \mathrm{MeV}$; see Fig. 11. In the experiment, the total charge was $60 \mathrm{pC}$, resulting in $\sim 420 \mathrm{fC}$ per beamlet. The three solenoids depicted in Fig. 11 were controlled independently via unipolar power supplies. We started with the normal operational configuration where the bucking and focusing solenoids had opposite polarities which yields relatively low magnetization of the beam. The bucking 


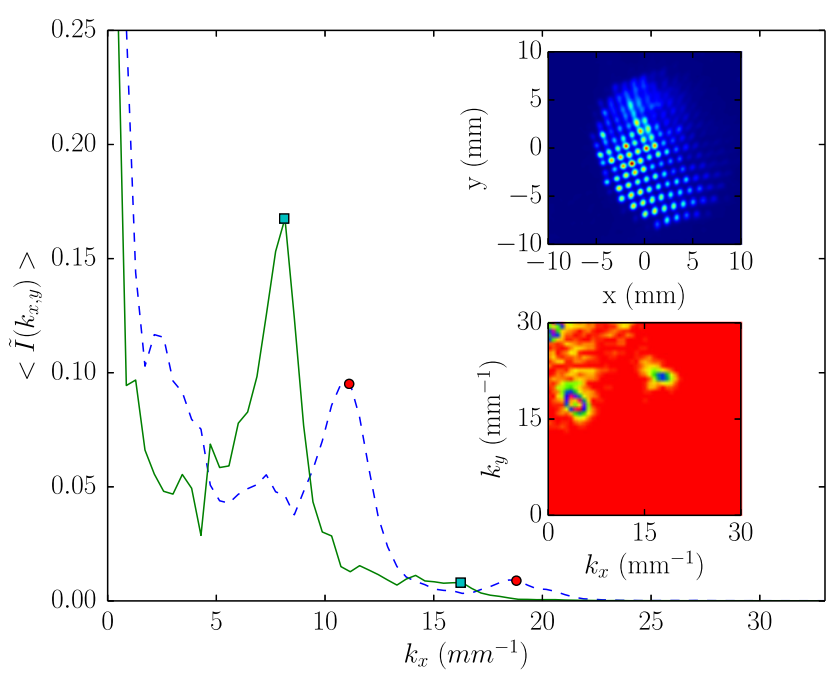

FIG. 12. TBF as a function of horizontal $k_{x}$ (solid trace) and vertical $k_{y}$ (dashed trace) spatial frequency. The pattern rotation angle is computed as $\tan \theta=k_{x_{1}} / k_{y_{1}}$. This data was extracted from the corresponding beam image (upper inset) and from projections of its 2D FFT image (lower inset).

solenoid current was slowly decreased to $0 \mathrm{~A}$ and the induced rotation of the beamlet formation was observed at YAG1 and YAG2 locations. Then the polarity of the bucking and focusing solenoids was flipped and the bucking solenoid current was ramped to $-500 \mathrm{~A}$. A total of 20 bucking solenoid current values were used to reach the maximum field at the cathode surface of $\simeq 1400 \mathrm{G}$. The value of $\psi$ computed between two screens (YAG1 and YAG2) increases with the magnetization. The full-width half-maximum (FWHM) sizes of the peaks in Fig. 12 were then accounted as error bars of the measurement. The

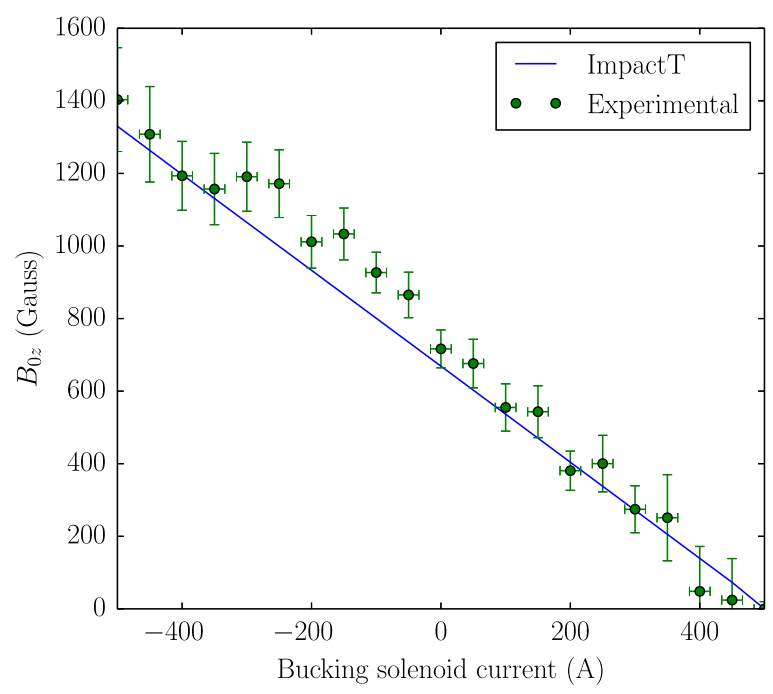

FIG. 13. Comparison between IMPACT-T simulations (solid blue line) and experimentally recovered values of $B_{0 z}$ (symbols with error bars). resulting values of $\psi$ were obtained from the $2 \mathrm{D}$ FFT technique detailed in Sec. II, and the MAM was recovered via Eq. (9). The values of the $B_{0 z}$ retrieved for different currents of the bucking solenoid are reported in Fig. 13. They appear to be in a very good agreement with IMPACT-T simulations of the measurement with a model of the solenoids simulated with POISSON [25], proving the validity of the method.

\section{SUMMARY}

We proposed and demonstrated a simple method to control the transverse distribution of an ultraviolet laser pulse used in a photoemission electron source. The technique, which involves a pair of microlens arrays, enables the generation of electron beam with homogenized transverse distribution with an associated increase in beam quality. We also employed the setup to produce patterned electron beams consisting of a two-dimensional array of electron beamlets. We demonstrated an application of patterned beams to the measurement of canonical angular momentum associated to a magnetized beam. We expect patterned beams to have a broad range of applications beyond beam-based diagnostics. A possible application at AWA is to combine the patterned beam with a transverseto-longitudinal phase-space-exchanging beam line and produce a bunch train with variable temporal spacing [26-32]. Additionally, given its simplicity, low cost and versatility we expect the present work to motivate further applications to photoemission electron sources and laserheater systems [33].

\section{ACKNOWLEDGMENTS}

This work was supported by the U.S. Department of Energy under Contract No. DE-SC0011831 with Northern Illinois University. The work by the AWA group is funded through the U.S. Department of Energy, Office of Science, under Contract No. DE-AC02-06CH11357. The work of A. H. and partially P.P. is supported by the U.S. Department of Energy under Contract No. DE-AC0207CH11359 with Fermi Research Alliance, LLC.

[1] S. Li, D. K. Bohler, W. J. Corbett, A. S. Fisher, S. Gilevich, Z. Huang, A. Li, D. F. Ratner, J. Robinson, and F. Zhou, LCLS injector laser modulation to improve FEL operation efficiency and performance, in Proceedings of the 2015 International Particle Accelerator Conference (IPAC15) (JACoW, Richmond, Virginia, 2015), TUPJE074.

[2] P. Schreiber, S. Kudaev, P. Dannberg, and U. D. Zeitner, Homogeneous led-illumination using microlens arrays, Proc. SPIE 5942, 59420K (2005).

[3] F. M. Dickey and S. C. Holswade, Laser Beam Shaping: Theory and Techniques, Optical Science and Engineering (Taylor \& Francis, London, 2000). 
[4] O. G. de Oliveira and D. W. de Lima Monteiro, Optimization of the Hartmann-Shack microlens array, Opt. Lasers Engg. 49, 521 (2011).

[5] Y. Shibata, T. Takahashi, T. Kanai, K. Ishi, M. Ikezawa, J. Ohkuma, S. Okuda, and T. Okada, Diagnostics of an electron beam of a linear accelerator using coherent transition radiation, Phys. Rev. E 50, 1479 (1994).

[6] W. S. Graves, F. X. Kärtner, D. E. Moncton, and P. Piot, Intense Superradiant X Rays from a Compact Source Using a Nanocathode Array and Emittance Exchange, Phys. Rev. Lett. 108, 263904 (2012).

[7] J. G. Power, M. E. Conde, W. Gai, Z. Li, and D. Mihalcea, Upgrade of the drive LINAC for the AWA facility dielectric two-beam accelerator, in Proceedings of the International Particle Accelerator Conference, Kyoto, Japan (ICR, Kyoto, 2010), THPD016.

[8] O. Chubar, A. Fluerasu, L. Berman, K. Kaznatcheev, and L. Wiegart, Wavefront propagation simulations for beam lines and experiments with SRW, J. Phys. Conf. Ser. 425, 162001 (2013).

[9] SUSS MicroOptics techinfo sheet 10, Beam homogenizing, 2008.

[10] We note another popular technique to quantify the quality of an optical beam relies on the decomposition into Zernike's polynomials. Our choice to use the twodimensional Fourier transform was motivated by the need to use one figure of merit to quantify both the quality of the homogenized beam and to also parametrize the modulated beam.

[11] T. E. Oliphant, Python for Scientific Computing, Comput. Sci. Eng. 9, 10 (2007).

[12] S. B. van der Geer et al., 3D space-charge model for GPT simulations of high brightness electron bunches, Inst. Phys. Conf. Ser. 175, 101 (2005).

[13] F. Zhou, I. Ben-Zvi, M. Babzien, X. Y. Chang, A. Doyuran, R. Malone, X. J. Wang, and V. Yakimenko, Experimental characterization of emittance growth induced by the nonuniform transverse laser distribution in a photoinjector, Phys. Rev. ST Accel. Beams 5, 094203 (2002).

[14] M. Rihaoui, P. Piot, J. G. Power, Z. Yusof, and W. Gai, Observation and simulation of space-charge effects in a radio-frequency photoinjector using a transverse multibeamlet distribution, Phys. Rev. ST Accel. Beams 12, 124201 (2009).

[15] K. Flottmann, Report No. DESY-TESLA-FEL-97-01, 1997, report available from DESY Hamburg Germany.

[16] J. Qiang, Report No. LBNL-62326, 2007, report available from BerkeleyLab.

[17] J. Barnes and P. Hut, A hierarchical $\mathrm{O}(\mathrm{N} \log \mathrm{N})$ forcecalculation algorithm, Nature (London) 324, 446 (1986).

[18] J. M. Maxson, I. V. Bazarov, W. Wan, H. A. Padmore, and C. E. Coleman-Smith, Fundamental photoemission brightness limit from disorder induced heating, New J. Phys. 15, 103024 (2013).

[19] A. Halavanau and P. Piot, Simulation of a cascaded longitudinal space charge amplifier for coherent radiation generation, Nucl. Instrum. Methods Phys. Res., Sect. A 819, 144 (2016).

[20] G. Ha, M. H. Cho, W. Namkung, J. G. Power, D. S. Doran, E. E. Wisniewski, M. Conde, W. Gai, W. Liu, C. Whiteford, Q. Gao, K.-J. Kim, A. Zholents, Y.-E Sun, C. Jing, and P. Piot, Precision Control of The Electron Longitudinal Bunch Shape Using an Emittance-Exchange Beam Line, Phys. Rev. Lett. 118, 104801 (2017).

[21] G. Qiang, C. Jing, S. Antipov, J. Shi, H. Chen, W Gai, J. Power, M. Conde, C. Whiteford, E. Wisniewski, W. Liu, and D. Doran, Transformer ratio enhancement experiment based on emittance exchanger in Argonne Wakefield Accelerator, in Proceedings of the 2016 Northern American Particle Accelerator Conference (NAPAC16), JACoW, Chicago, IL (to be published), THPOA08.

[22] M. Reiser, Theory and Design of Charged Particle Beams (Wiley, New York, 1995).

[23] A similar method was discussed in Ref. [24] for the case of slits located downstream of the electron source.

[24] Y. E Sun, P. Piot, K. J. Kim, N. Barov, S. Lidia, J. Santucci, R. Tikhoplav, and J. Wennerberg, Generation of angularmomentum-dominated electron beams from a photoinjector, Phys. Rev. ST Accel. Beams 7, 123501 (2004).

[25] J. H. Billen and L. M. Young, in Proceedings of the 1993 Particle Accelerator Conference, Washington, DC (IEEE, Piscataway, NJ, 1993), pp. 790-792.

[26] V. Marx, Neurobiology: Brain mapping in high resolution, Nature (London) 503, 147 (2013).

[27] Y. Yoshida, H. Kashima, K. Kan, K. Norizawa, A. Ogata, S. Tagawa, T. Kondoh, and J. Yang, Spatial resolution and contrast of the intensity modulated electron beam by the photocathode rf gun for the radiation therapy, in Proceedings of the 11th European Particle Accelerator Conference, Genoa, 2008 (EPS-AG, Genoa, Italy, 2008), p. 1809.

[28] P. Piot, Y.-E Sun, J. G. Power, and M. Rihaoui, Generation of relativistic electron bunches with arbitrary current distribution via transverse-to-longitudinal phase space exchange, Phys. Rev. ST Accel. Beams 14, 022801 (2011).

[29] Y.-E Sun, P. Piot, A. Johnson, A. H. Lumpkin, T. J. Maxwell, J. Ruan, and R. Thurman-Keup, Tunable Subpicosecond Electron-Bunch-Train Generation Using a Transverse-to-Longitudinal Phase-Space Exchange Technique, Phys. Rev. Lett. 105, 234801 (2010).

[30] Y.E. Sun, J. G. Power, K. J. Kim, P. Piot, and M. M. Rihaoui, Design study of a transverse-to-longitudinal emittance exchange proof-of-principle experiment, in Proceedings of the 22nd Particle Accelerator Conference, PAC-2007, Albuquerque, NM (IEEE, New York, 2007), pp. 3441-3443.

[31] R. Brinkmann, Y. Derbenev, and K. Flottmann, A low emittance, flat-beam electron source for linear colliders, Phys. Rev. ST Accel. Beams 4, 053501 (2001).

[32] K. J. Kim, Round-to-flat transformation of angularmomentum-dominated beams, Phys. Rev. ST Accel. Beams 6, 104002 (2003).

[33] D. Ratner (private communication). 